\title{
Game-theoretic model of wide social groups' behavior with stimulation of volunteering activities
}

\author{
M I Geraskin ${ }^{1}$ \\ ${ }^{1}$ Samara National Research University, Moskovskoe Shosse, 34, Samara, Russia, 443086 \\ e-mail: innovation@ssau.ru
}

\begin{abstract}
The problem of developing tools for the stimulation system of socially optimal actions (volunteering) is considered. Based on the study of the population's differentiation according to the propensity to an altruism, the game-theoretic model of the social group's behavior is formed, accounting for the incentives for volunteering. In the cases of the linear decreasing incentive function and the linear cost functions of agents, the Cournot-Nash equilibrium mechanism in the corresponding game is proved. An existence of the equilibrium actions and an impact of incentives on the volunteers' time distribution are confirmed by the simulation of the volunteers' behavior in Russia.
\end{abstract}

\section{Introduction}

In Russia, in recent years, volunteering has been developing, because in 2016 a number of the volunteers was 1.435 million $^{1}$, i.e., $1 \%$ of the population. The volunteering is realized in the performance of actions that maximize the collective utility function, that is, socially optimal actions. The volunteers do not receive income, therefore, these actions do not correspond to the criterion of individual rationality. The Russian statistics demonstrates that moral motivation is effective for a narrow social group of altruistic people. Among the broad masses of the population, trends of individual rationalism [1,2] are exist. The state programs [3,4] implement for overcoming of these trends. In addition, the expansion of volunteering can be provided by the system of stimulation of these actions on the base of the state information system [5].

The interconnection algorithms of the stimulation system and the information system [6,7] enable to solve the problems of personalized registration of citizens' actions, the distribution of the state incentive fund, the monitoring of the of the stimulation effectiveness.

The methodological basis for the development of incentive systems includes the following mechanisms: the competitive mechanism is Pareto-efficient and optimal by the criterion of the additive utility function under non-coalition [8] and coalition [9] agents' behavior; the mechanism of sequential resource distribution (MSRD) [10,11]; the mechanisms of direct and reverse priorities [12]. For MSRD the existence and the uniqueness were proved [13]; according to MSRD, the incentive is distributed [14] as a minimum of the agent's message about its action and the average undistributed rest of incentives. MSRD satisfies [15] the conditions of individual rationality, Pareto efficiency and

\footnotetext{
${ }^{1}$ Labor and employment in Russia 2017: Statistic compilation / Rosstat. Moscow. 2017. 261 p. http://www.gks.ru/free_doc/doc_2017/ trud_2017.pdf
} 
non-manipulability. MSRD is not applicable in a system with independent and simultaneous agents' actions, because the MSRD implies the sequential registration of the agent' actions and the distribution of incentives. Therefore, we use the compensatory linearly decreasing stimulation function, for which these conditions were proved [6,7]. The simulation of the social management $[16,17]$ on the basis of the large groups of population demonstrated the effectiveness of this approach. The utility functions of agents can take into account the symmetry and asymmetry of their awareness [18], which is provided by analyzing the correlation of information flows between social groups $[19,20]$.

The stimulation system of socially optimal actions provides the following results:

- an increase in a number of the volunteers and the time fund of these actions;

- the cross-impact of the altruism and the individual rationality on the behavior of the population groups;

- an emergence of the contradictions between the interests of large social groups, differentiated by a degree of the propensity to the altruism.

Consequently, the model of the population behavior can be formed as the non-cooperative game of the social groups (hereinafter, the agents). The utility functions of these agents include both the incentives for performing socially optimal actions and the loss of the income due to the redistribution of available time, which is a constraint.

\section{Methods}

We assume that, for the $k$ th agent, a continuous function $\zeta_{k}(\bullet)$ is defined, and we denote the socially optimal actions time interval in the absence of stimulation as $a_{k}$. The function $\zeta_{k}(\bullet)$ expresses the dependence of $a_{k}$ on the available time fund, and the function has the following form:

$$
a_{k}=\zeta_{k}(D)=D^{\delta_{a k}}, \delta_{a k} \in[0,1], D>>1, k \in K,
$$

where $D$ is the disposable time fund, i.e., the physical time fund with the exception of the rest time; $\delta_{a}$ is the elasticity coefficient of the "charitable" time to $D$, which characterizes the propensity to the altruism; $K$ is the set of agents; $a_{k}$ is the component of the socially optimal actions vector $\mathbf{A}=\left\{a_{k}, k \in K\right\}$.

Definition: the altruism (the propensity to the charity) of the kth agent is called the type of agent, for which $\delta_{a k}>0,5$ in function (1).

We introduce the hypothesis of the influence of the propensity to the altruism on the agent's behavior: an increase in the propensity to the altruism leads to a decrease in the utility of the wage, i.e.,

$$
U_{\delta_{a}}^{\prime}\left(p_{d}\right)<0,
$$

where $d$ is the working time interval; $p_{d}$ is the price (the tariff rate) of the working time; $U(\bullet)$ is the continuously differentiated utility function of the agent.

In the case of the stimulation, the model of the agents' actions choice includes the utility function and the stimulation function. We describe these components of the model.

The agent's utility function is the difference between the sum of the incentives and the costs of the working time loss:

$$
U_{k}\left(a_{k}\right)=p_{a} a_{k}-p_{d}^{1-\delta_{a k}} a_{k}, k \in K,
$$

where $p_{a}$ is the sum of the insensitive, i.e., the price of a unit of "charitable" time. Formula (3) corresponds to hypothesis (2), because, with a growth of $\delta_{a}$, the influence of the working time price and the working time fund on the agent's utility decreases.

The stimulation function determines the price of the insensitive as follows [7]:

$$
p_{a}(\mathrm{~A})=b_{1}-b_{2} \sum_{k \in K} n_{k} a_{k}, k \in K, b_{1}, b_{2}>0,
$$


where $n_{k}$ is the number of agents in $k$ th social group, which is constant in the current period; $b_{1}, b_{2}$ are the constant coefficients, calculated by the following formulas

$$
b_{1}=p_{d} \frac{A^{0}}{A^{0}-A^{D}}, b_{2}=\frac{p_{d}}{A^{0}-A^{D}}, A^{0}=\sum_{k \in K} a_{k}^{0}, A^{D}=\frac{D}{2} \sum_{k \in K} n_{k} .
$$

The coefficients $b_{1}, b_{2}$ depend on the of agents' actions vector in the previous period $\mathbf{A}^{0}=\left\{a_{k}^{0}, k \in K\right\}$. Formulas (4a) are obtained on the basis of the following conditions ${ }^{1}: 1$ ) in the absence of incentives (i.e., for $p_{a}(\mathrm{~A})=0$ ), the total number of the socially optimal actions is equal to $\left.\sum_{k \in K} a_{k}^{0} ; 2\right)$ when the price of the incentive is equal to the average wage $p_{d}$, the disposable time fund is divided equally between the working time and the "charitable” time (i.e., $\frac{D}{2}$ ).

We consider the problem of searching for the Nash equilibrium vector $\mathbf{A}$ from the maximum of the utility function (3) under condition (4) in the case of the constancy of the agents' number in all social groups (i.e., $\frac{\partial n_{k}}{\partial p_{a}}=0 \forall k \in K$ ).

\section{Results and discussion}

The equilibrium conditions are formulated as the following assertion.

Assertion $1^{2}$. The actions vector $A$, satisfying the following conditions

$$
\begin{aligned}
& b_{1}-b_{2} \sum_{j \in K} n_{j} a_{j}-b_{2} n_{k} a_{k}\left(1+\sum_{j \in K \backslash k} \frac{\partial a_{j}}{\partial a_{k}}\right)-p_{d}^{1-\delta_{a k}}=0, \\
& \sum_{j \in K \backslash k} \frac{\partial a_{j}}{\partial a_{k}}>-2, k \in K,
\end{aligned}
$$

is the Nash equilibrium in problem (3), (4).

In the case of the Cournot hypothesis [21], all agents symmetrically do not change the selected actions in response to the environment's actions, i.e.

$$
\frac{\partial a_{j}}{\partial a_{k}}=0, j \in K \backslash k,
$$

which corresponds to the simultaneous and independent choice of actions.

Under condition (7), system (5) has the following form

$$
2 n_{k} a_{k}+\sum_{j \in K \backslash k} n_{j} a_{j}-\alpha_{k}=0, \alpha_{k}=\frac{b_{1}+p_{d}^{1-\delta_{a k}}}{b_{2}}, k \in K,
$$

The solution of the system (8) by the Kramer's method can be written as follows:

$$
a_{k}^{*}=\frac{n \alpha_{k}-\sum_{j \in K \backslash k} \alpha_{j}}{(n+1) n_{k}}, k \in K,
$$

where the symbol "*” denotes the equilibrium values, $n$ is the number of agents in the system. For the Cournot-Nash equilibrium (9), conditions (6) are satisfied considering (7).

\footnotetext{
${ }^{1}$ In the case of these conditions, the system of equations $b_{1}-b_{2} A^{0}=0, b_{1}-b_{2} A^{D}=p_{d}$ leads to the solution (4a).

${ }^{2}$ Proof of assertion 1. Function (3) under (4) has the form $U_{k}\left(a_{k}\right)=\left(b_{1}-b_{2} \sum_{k \in K} n_{k} a_{k}\right) a_{k}-p_{d}^{1-\delta_{a k}} a_{k}$. Therefore, the extremum necessary condition $U_{a_{k}}^{\prime}=0$ can be written in the form (5). The sufficient maximum condition $U_{a_{k}}^{\prime \prime}<0$ leads to (6).
} 
We simulate the equilibrium (11) using the example of the social groups of Russian volunteers (Table 1). The actual propensity to the altruism is calculated by the formula $\delta_{a k}(a)=\frac{\ln a_{k}}{\ln D}$ that follows from formula (1), where the weekly time fund is taken equal to $D=112$ hours; the graph of the function $\delta_{a k}(a)$ is shown in Fig. 1. The actual distribution function of the population in groups with different propensities to the altruism is calculated by the formula $f_{k}(a)=\frac{n_{k}}{\sum_{k \in K} n_{k}}$.

Table 1 Analysis of the volunteers in 2016.

\begin{tabular}{|c|c|c|c|c|c|c|c|c|c|c|}
\hline \multirow[b]{2}{*}{ Indicator } & \multirow[b]{2}{*}{ Total } & \multicolumn{9}{|c|}{ Including time worked per week, hours } \\
\hline & & $<9$ & $9-15$ & $16-20$ & $21-30$ & $31-40$ & $41-50$ & & $>51$ & \\
\hline The average & & & & & & & & & & \\
\hline $\begin{array}{l}\text { "charitable" time } a \text {, } \\
\text { hours }\end{array}$ & 8.6 & 2.35 & 15 & 20 & 30 & 40 & 50 & 60 & 70 & 80 \\
\hline $\begin{array}{l}\text { The population } n_{k} \text {, } \\
\text { thousand }\end{array}$ & 1435 & 997 & 243 & 82 & 48 & 23 & 11 & 9.8 & 9 & 8 \\
\hline $\begin{array}{l}\text { The total } \\
\text { "charitable" time } A \text {, } \\
\text { thousand hours }\end{array}$ & 12398 & 2343 & 3645 & 1640 & 1440 & 920 & 550 & 588 & 630 & 640 \\
\hline $\begin{array}{l}\text { The structure of } \\
\text { "charitable" time, \% }\end{array}$ & 100 & 18.90 & 29.40 & 13.23 & 11.62 & 7.42 & 4.44 & 4.74 & 5.08 & 5.16 \\
\hline$\delta_{a k}(a)$ & 0.181 & 0.181 & 0.574 & 0.635 & 0.721 & 0.782 & 0.829 & 0.868 & 0.900 & 0.929 \\
\hline$f_{k}(a)$ & & 0.070 & 0.018 & 0.005 & 0.000 & 0.000 & 0.000 & 0.000 & 0.000 & 0.000 \\
\hline The agent's index & & 1 & 2 & 3 & 4 & 5 & 6 & 7 & 8 & 9 \\
\hline
\end{tabular}

The model of the probability distribution of volunteers according to the "charitable" time has the form of the normal law with certain values of the kurtosis and the asymmetry [7]:

$$
f(a)=\frac{1}{\sigma_{a} \sqrt{2 \pi}} e^{-\frac{w\left(a-\bar{a}^{l}\right)^{2}}{2 \sigma_{a}^{2}}},
$$

where $\bar{a}, \sigma_{a}$ are the mathematical expectation and the standard deviation of the initial distribution of the random variable of the "charitable" time; $l$ is the coefficient taking into account the asymmetry ( $l>1$ is the left asymmetry, $l<1$ is the right asymmetry) in comparison with the normal law $(l=1)$; $w$ is the coefficient taking into account the kurtosis ( $w<1$ is a more uniform distribution, $w>1$ is a less uniform distribution) in comparison with the normal law $(w=1)$.

According to a degree of the propensity to the altruism, the model of the distribution density of volunteers has a similar form (10), but because the function $\delta_{a k}\left(a_{k}\right)$ is calculated through a logarithmic relationship, instead of formula (10), the following formula is used:

$$
f\left(\delta_{a}\right)=\frac{1}{e^{\sigma_{\delta}} \sqrt{2 \pi}} e^{-\frac{e^{m_{1}}\left(e^{\delta_{a}}-\left(e^{\bar{\delta}}\right)^{k^{1}}\right)^{2}}{2\left(e^{\sigma_{\delta}}\right)^{2}}},
$$

where $\bar{\delta}, \sigma_{\delta}$ are the mathematical expectation and the standard deviation of the initial distribution of the random value of the propensity to the altruism, the parameters $w_{1}, l_{1}$ are similar to the parameters $w, l$ for the distribution (10).

Using the least squares algorithm implemented in the MSExcel processor, the following values of the coefficients of functions (10), (11) are obtained: $\bar{a}=8,6, \sigma_{a}=0,57, w=0,007, l=0,58$, $\bar{\delta}=0,18, \sigma_{\delta}=-0,65, w_{1}=0,43, l_{1}=0,08$. The statistical estimates of regressions (for function (10) 
$R^{2}=0,99, F=257$, for function (11) $R^{2}=0,99, F=218$ ) prove their adequacy. Therefore, the distributions of volunteers according to the time and the propensity to the altruism correspond to the right branch of the Gauss function, that is, the mathematical expectations of the time and the propensity to the altruism are close to the minimum of these indicators. Functions (10), (11) with regard to the indicated coefficients are shown in Fig. 1.2.

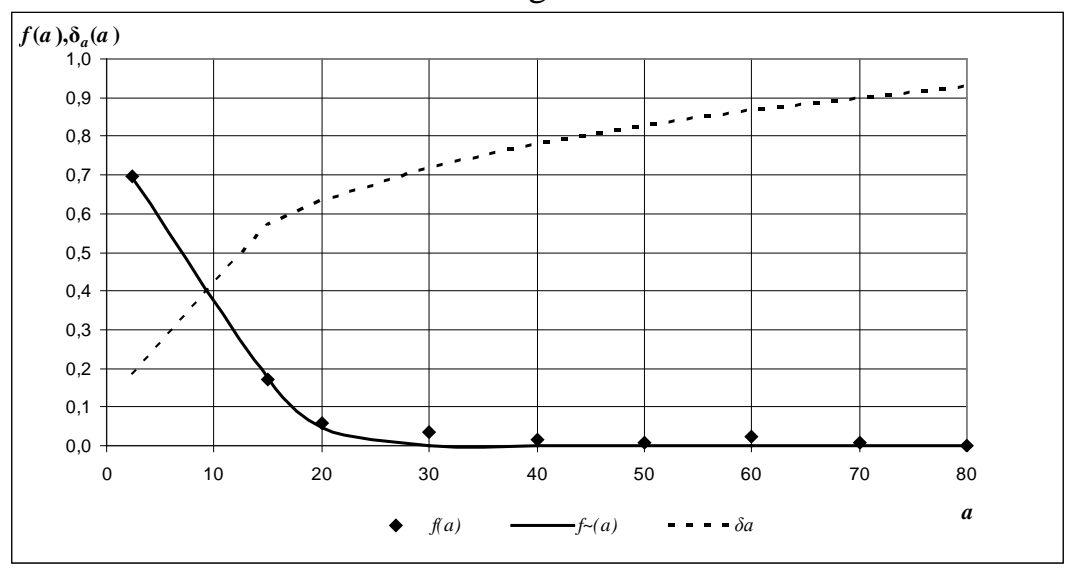

Figure 1. Density distribution function of volunteers by the time and the propensity to altruism.

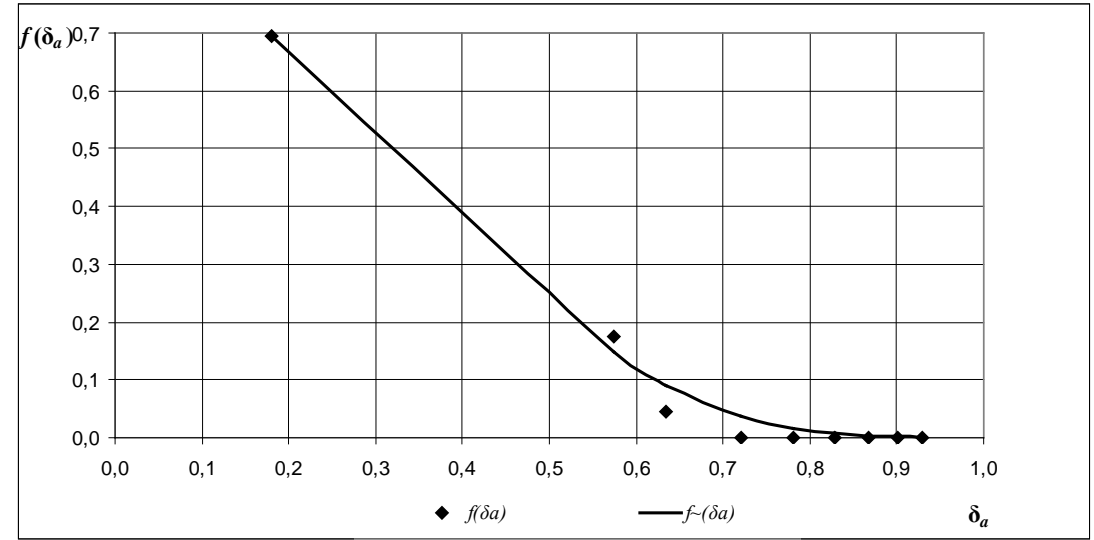

Figure 2. The density function of the distribution of volunteers by the degree of propensity to altruism.

Consider the Cournot-Nash equilibrium (9) for agents, which are indexed in accordance with Table. 1. In Fig. 3 the distribution functions of the time depending on a degree of the propensity to the altruism with different insensitives are shown. In Fig. 4 the dependence of the volunteers' time on the insensitive is demonstrated.

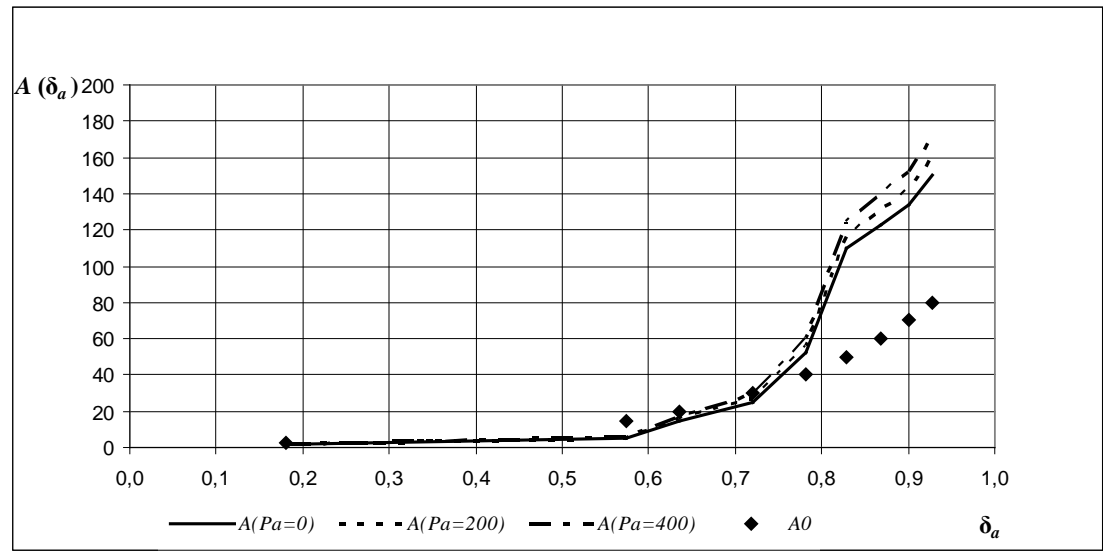

Figure 3. The distribution of the volunteers' time depending on the insensitive. 


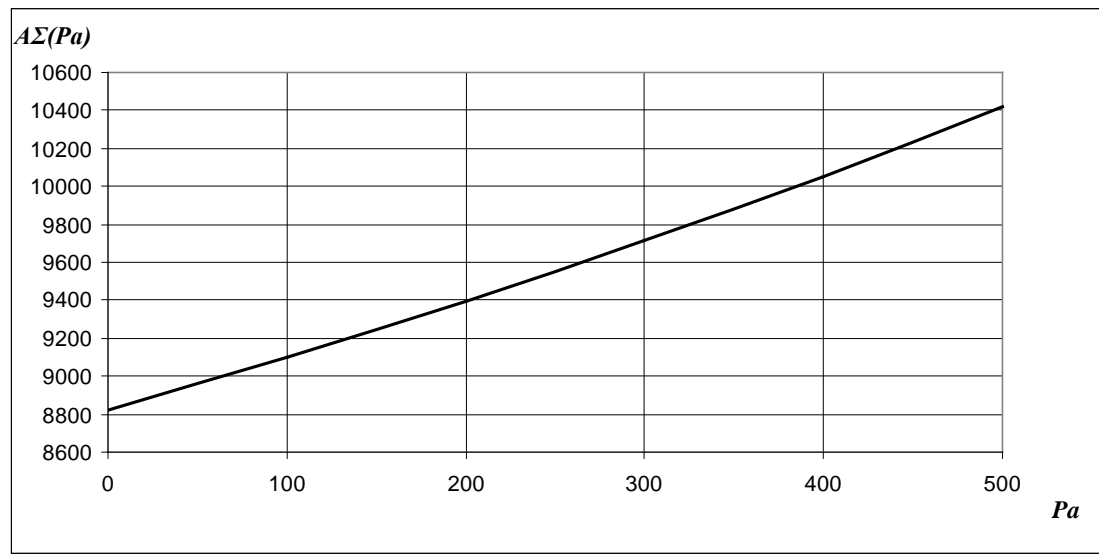

Figure 4. The dependence of the total volunteers' time on the insensitive.

The analysis of the Cournot-Nash equilibria simulation leads to the following conclusions.

First, the distribution of "charitable" time is closest to the real distribution for $P a=0$, but the calculated equilibrium actions are lower than the actual values for the agents with low propensity to the altruism, and higher than the actual values for the agents with high propensity to the altruism. Moreover, the volunteers' time at $P a=0$ is lower than the actual value. However, the average deviation of the total time for each agent from the total actual time is $4 \%$. Consequently, in the developed equilibrium model, the influence of the population's propensity to the altruism on the volunteers' time is slightly exaggerated.

Second, in the case of the equilibrium distribution, the agents with the highest propensity to the altruism make the greatest contribution to the volunteers' time.

Third, with a growth of the insensitive, the distribution of "charitable" time becomes increasingly uneven, that is, the "charitable" time increases slightly for the agents with low propensity to the altruism and increases sharply for the agents with high propensity to the altruism. Therefore, the agents with high propensity to the are the most sensitive to stimulation.

Fourth, with an increasing in the incentive, the total "charitable" time grows, i.e., the stimulation influences on the involvement in volunteering for high and low altruistic agents.

\section{Conclusion}

The problem of the developing the information tools for the system of the volunteering stimulation is considered. In the article, the following main results are obtained.

The game-theoretic model of the social groups' behavior is developed, taking into account the stimulation of volunteering, based on the differentiation of the population according to the altruism and the individual rationality.

In the case of the linear diminishing incentive function and the linear agents' cost functions, the Cournot-Nash equilibrium mechanism in the corresponding game is proved.

The simulation of the volunteers' behavior in Russia demonstrates the adequacy of the model, the existence of the equilibrium actions vector and the effect of the stimulation on the volunteers' time.

The analysis shows that the stimulation system has the greatest effect on the agents with high propensity to altruism, but the growth of socially optimal actions is manifested in all social groups.

\section{Literature}

[1] Roland G 2000 Transition and Economics. Politics, Markets, and Firms (Cambridge: MIT Press) p 840

[2] Braguinsky S, Yavlinsky G 2000 Incentives and Institutions. Transition to a Market Economy in Russia (NJ.: Princeton University Press) p 420

[3] RF Government Decree of 30.12.2015 N 1493 "On State program" Patriotic Education of Citizens of the Russian Federation for 2016-2020" 
[4] RF Government Decree of December 27, 2012 N 2567-r "On the state program of the Russian Federation" Development of Culture and Tourism "2013-2020"

[5] RF Government Decree of 15.04.2014 N 313 (as amended on 10.21.2016.) "On approval of the Russian Federation, the state program" Information Society (2011-2020)"

[6] Geraskin M I 2017 Algorithms of the information stimulation system of Russian citizens' sociooptimal actions CEUR Workshop Proceedings 1903 92-99

[7] Geraskin M I 2018 Analysis of the influence of citizens' altruism on the effectiveness of the socially-optimal actions stimulation system CEUR Workshop Proceedings 2212 431-439

[8] Burkov V N, Danev B, Enaleev A K, Nanev T B, Podvalny L D and Yusupov B S 1988 Competitive mechanisms in problems of distribution of scarce resources Avtomatika $i$ telemekhanika 11 142-153

[9] Burkov V N, Enaleev A K and Kalenchuk V F 1989 Coalition with the competitive mechanism of resource distribution Avtomatika i telemekhanika 12 81-90

[10] Burkov V N, Enaleev A K and Lavrov Y G 1992 Synthesis of optimal planning and incentive mechanisms in the active system Avtomatika i telemekhanika 10 113-120

[11] Burkov V N, Iskakov M B and Korgin N A 2010 Application of generalized median schemes for the construction of non-manipulable mechanism multicriterion active expertise Automation and Remote Control 71(8) 1681-1694

[12] Korgin N A 2009 Equivalence of non-manipulable and non-anonymous priority resource distribution mechanisms Upravleniye bol'shimi sistemami 26.1 319-347

[13] Burkov V N, Gorgidze I I, Novikov D A and Yusupov B S 1997 Models and cost and revenue distribution mechanisms in the market economy (Moskva: Institut problem upravleniya) p 356

[14] Korgin N A 2010 Use of intersection property for analysis of feasibility of multicriteria expertise results Automation and Remote Control 71(6) 1169-1183

[15] Burkov V N, Korgin N A and Novikov D A 2016 Problems of aggregation and decomposition mechanisms of management of organizational and technical systems Problemy upravleniya 5 $14-23$

[16] Khaimovich I N, Ramzaev V M and Chumak V G 2016 Use of big data technology in public and municipal management CEUR Workshop Proceedings 1638 864-872

[17] Khaimovich I N, Ramzaev V M and Chumak V G 2015 Challenges of data access in economic research based on Big Data technology CEUR Workshop Proceedings 1490 327-337

[18] Faizliev A R, Korotkovskaya E V, Sidorov S P, Smolov F M and Vlasov A A 2018 Utility Maximization for an Investor with Asymmetric Attitude to Gains and Losses over the MeanVariance Efficient Frontier Journal of Physics: Conference Series 1141(1) 012017

[19] Sidorov S, Faizliev A and Balash V 2017 Measuring long-range correlations in news flow intensity time series International Journal of Modern Physics C 28(8) 1750103

[20] Kulikovskikh I M 2017 Anomaly detection in an ecological feature space to improve the accuracy of human activity identification in buildings Computer Optics 41(1) 126-133 DOI: 10.18287/2412-6179-2017-41-1-126-133

[21] Cournot A A 1960 Researches into the Mathematical Principles of the Theory of Wealth (London: Hafner) 